
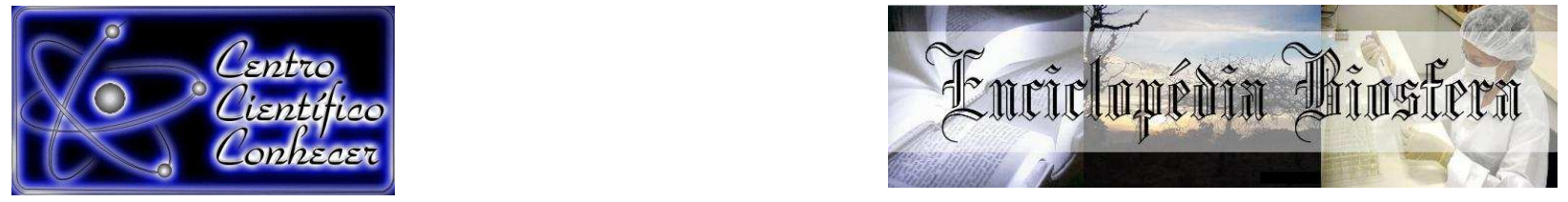

\title{
VIOLÊNCIA CONTRA A MULHER NO PARANÁ: ASPECTOS EPIDEMIOLÓGICOS
}

\author{
Fernanda Shizue Nishida ${ }^{1}$; Vivian Carla de Castro ${ }^{2}$.
}

${ }^{1}$ Enfermeira, doutora em Ciências pela Universidade de São Paulo, professora do Programa de Mestrado em Promoção da Saúde do Centro Universitário de Maringá - UNICESUMAR; Pesquisadora do Instituto Cesumar de Ciência, Tecnologia e Inovação (ICETI) - Maringá,

Paraná, Brasil. fernanda.nishida@unicesumar.edu.br

${ }^{2}$ Enfermeira, doutoranda em Enfermagem pela Universidade Estadual de Maringá Maringá, Paraná, Brasil.

Recebido em: 03/10/2016 - Aprovado em: 21/11/2016 - Publicado em: 05/12/2016 DOI: 10.18677/EnciBio_2016B_140

\begin{abstract}
RESUMO
A violência contra a mulher é um relevante problema de saúde pública bem como uma violação aos direitos humanos. O objetivo deste estudo foi caracterizar o perfil epidemiológico das notificações de violência contra a mulher, ocorridas no Paraná entre 2009-2014. Estudo transversal, descritivo de abordagem quantitativa que utilizou dados do Sistema de Informação de Agravos de Notificação (SINAN) do Departamento de Informática do SUS (DATASUS), do Ministério da Saúde. A população de estudo foi constituída por todas as 22.362 notificações de violência contra mulheres residentes no estado do Paraná. Foram incluídas mulheres entre 15-59 anos. Os resultados encontrados permitiram verificar que a violência contra a mulher é elevada e apresentou aumento até o ano de 2013, ocorre em maiores proporções em mulheres brancas de baixa escolaridade, com idade entre 20-29 anos. O local de ocorrência mais comum foi o domicílio, tendo o cônjuge como principal agressor e a violência física a forma mais prevalente. O principal desfecho observado foi o encaminhamento ambulatorial. Conclui-se que a violência contra a mulher é um crescente problema, sua magnitude evidencia a desigualdade de gênero e a emergente necessidade da elaboração de políticas públicas para redução, controle do problema, proteção das mulheres em situação de vulnerabilidade e promoção da saúde e qualidade de vida dessas.
\end{abstract}

PALAVRAS-CHAVE: Epidemiologia, Saúde da Mulher, Violência contra a Mulher.

\section{VIOLENCE AGAINST WOMEN IN PARANÁ: EPIDEMIOLOGICAL ASPECTS}

\begin{abstract}
Violence against women is a major public health problem and a violation of human rights. The aim of this study was to characterize the epidemiological profile of reports of violence against women occurred in Paraná between 2009-2014. cross-sectional, descriptive study of quantitative approach used data from the Notifiable Diseases Information System (SINAN) of the Department of SUS (DATASUS), the Ministry of Health. The study population consisted of all 22,362 reports of violence against women living in the state of Paraná. Women were included between 15-59 years. The data revealed that violence against women is high and it increased until the year 2013, occurs in higher proportions among white women with low educational level, aged 20-29 years. The most common occurrence site was the home, and the spouse as the primary aggressor and physical violence the most prevalent form. The primary ENCICLOPÉDIA BIOSFERA, Centro Científico Conhecer - Goiânia, v.13 n.24; p. 15162016
\end{abstract}


outcome was observed outpatient referral. It is concluded that violence against women is a growing problem, its magnitude shows gender inequality and the emerging need to develop public policies to reduce, problem control, women's protection in vulnerable situations and promote the health and quality of life of these.

KEYWORDS: Women's Health, Violence Against Women, Epidemiology.

\section{INTRODUÇÃO}

De acordo com a Organização Mundial da Saúde (OMS) a violência contra a mulher é um relevante problema de saúde pública bem como uma violação aos direitos humanos. A violência contra esse grupo ocorre de forma generalizada em todo o mundo, se constituindo em um agravo de proporções epidêmicas que requer ações urgentes. Todo indivíduo, seja mulher, homem ou criança tem direito a vida livre de violência (WHO, 2013).

A violência se constitui em um fenômeno histórico e social, apresentando distintos conteúdos e formas nas diversas sociedades, que varia de acordo com valores culturais que influenciam juízos éticos em cada uma delas. As diferenças culturais dificultam a conceituação da violência, todavia certos aspectos que a envolve são mantidos, nas diversas sociedades e culturas, permitindo sua caracterização (LUCENA et al., 2012).

O termo violência contra a mulher abrange muitas formas de violência, seja por parceiro ou não, e inclui violência física (sexual ou não), psicológica, crimes contra a honra, tráfico de mulheres entre outros (WHO, 2013). No ano de 2006, foi criada, no Brasil, a Lei n. 11.340, mais conhecida como Lei Maria da Penha, que estabeleceu mecanismos para coibir a violência doméstica e familiar contra a mulher (BRASIL, 2006). De acordo com o Instituto de Pesquisa Econômica e Aplicada (IPEA) anos após a lei ser sancionada há ainda uma lacuna acerca de estudos empíricos que avaliem o panorama da violência após a legislação (IPEA, 2015).

Estudo realizado por ACOSTA et al. (2013) aponta que a Lei Maria da Penha representou um importante avanço nas questões referentes aos direitos das mulheres, no entanto, a redução do número de agressões entre 2001 e 2010 foi pequena. Referem ainda que há dez anos, oito mulheres sofriam agressões a cada dois minutos, atualmente cinco ainda sofrem com a violência. Embora os resultados sejam positivos, muito ainda precisa ser feito para inibir a violência contra a mulher.

Segundo LUCENA et al. (2012) as consequências da violência, sofrida pela mulher, materializam-se em agravos biológicos, psicológicos, morais e sociais, que dificultam sua experiência de viver a igualdade humana e social. De acordo com SILVA et al. (2013) conhecer informações epidemiológicas sobre a violência contra a mulher são extremamente relevantes para 0 estabelecimento de políticas públicas, de prevenção à violência e de atendimento às vítimas. Neste contexto, o objetivo deste estudo foi caracterizar o perfil epidemiológico das notificações de violência contra a mulher ocorridas no Paraná entre 2009-2014.

\section{MATERIAL E MÉTODOS}

Estudo transversal, descritivo de abordagem quantitativa que utilizou dados do Sistema de Informação de Agravos de Notificação (SINAN) do Departamento de Informática do SUS (DATASUS), do Ministério da Saúde. A população de estudo foi constituída por todas as 22.362 notificações de violência contra mulheres residentes no estado do Paraná. Foram incluídas mulheres entre 15-59 anos. As variáveis de 
estudo foram: raça, escolaridade, tipo de violência (violência física, psicológica ou moral, tortura, violência sexual, espancamento, enforcamento), agressão com objetos contundentes, perfuro-cortantes, substância ou objeto quente, envenenamento, arma de fogo, ameaça, assédio sexual e estupro; quem foi o praticante da ação (pai, mãe, madrasta, padrasto, cônjuge, ex-cônjuge, namorado (a), ex-namorado (a), filho (a), irmão, amigo ou conhecido, cuidador, patrão, pessoa com relacionamento instável, policial ou agente da lei). Tipo de encaminhamento ao setor saúde e evolução dos casos.

Para a análise foram feitas distribuições percentuais para variáveis categóricas e taxa de variação anual entre início e fim do período estudado. Dispensou-se a submissão ao Comitê de Ética em Pesquisa envolvendo Seres Humanos por tratar-se de informações públicas. Segundo a Comissão Nacional de Ética em Pesquisa (CONEP) e Conselho Nacional de Saúde (CNS) pesquisas envolvendo apenas dados de domínio público que não identifiquem os participantes da pesquisa, sem envolvimento de seres humanos, não necessitam aprovação por parte do Sistema CEP-CONEP (http://conselho.saude.gov.br/).

\section{RESULTADOS E DISCUSSÃO}

O total de notificações no período foi de 22.362, sendo possível verificar um aumento ao longo dos anos. O aumento ocorreu até o ano de 2013 e após, há um declínio (Figura 1).

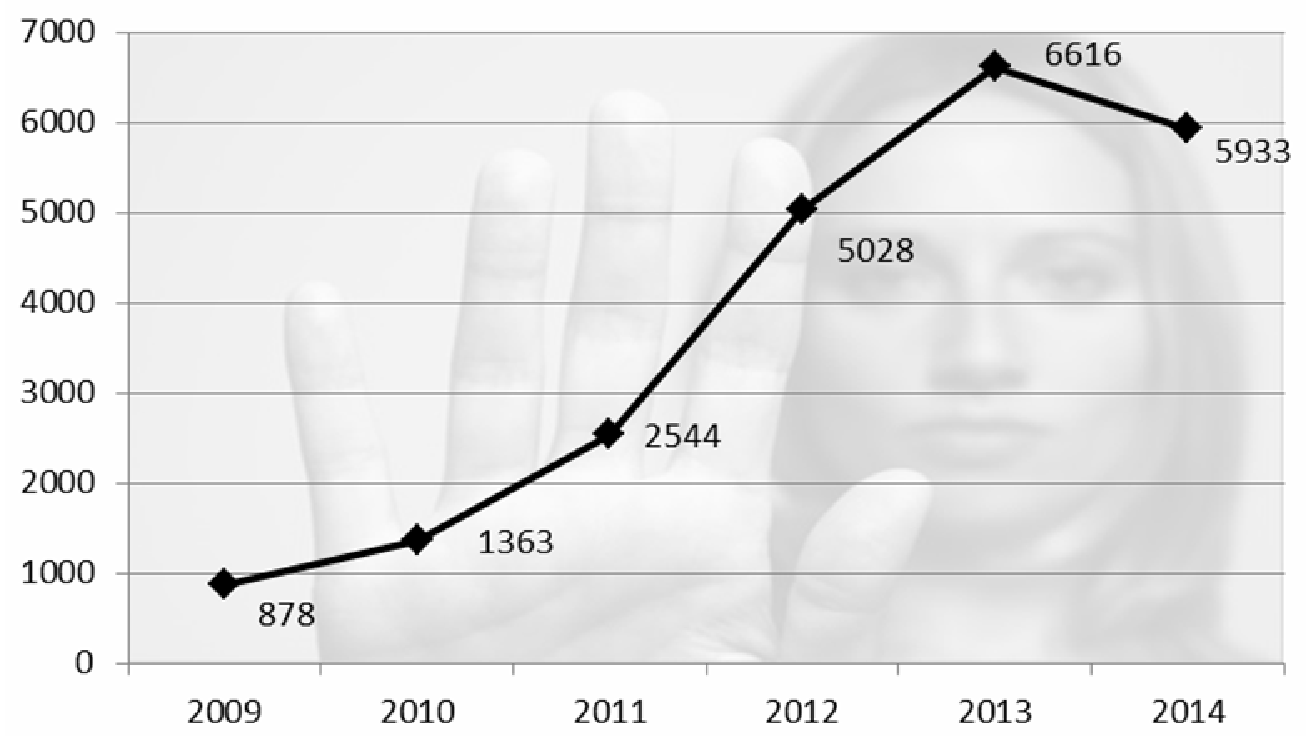

FIGURA 1. Distribuição das notificações de violência contra a mulher no Paraná 2009-2014. Fonte: SINAN, 2015.

Analisando a taxa de variação entre $2009-2013$ o aumento foi de $653,53 \%$. Já entre 2013-2014 ocorreu declínio de 10,32\% no total de notificações. A notificação configura-se como uma das estratégias primordiais do Ministério da Saúde no âmbito das ações contra a violência e como estratégia para articulação de políticas de saúde, sendo estabelecida como obrigatória, através da notificação compulsória de violência (VELOSO et al., 2013). Em Brasília, estudo verificou que entre 20092012 houve aumento no número de notificações (SILVA \& OLIVEIRA, 2016). Em Belém - PA pesquisadores verificaram que entre 2009-2011 o crescimento nas notificações de violência foi de $240 \%$ (VELOSO et al., 2013). Essas informações 
permitem refletir que o problema é persistente e que embora mecanismos como, a notificação e a legislação tenham sido criados para reduzir esse agravo, existem muitos obstáculos ainda a serem ultrapassados.

A faixa etária mais acometida foi de mulheres entre 20,29 anos com $28,45 \%$ (6805). As demais faixas foram entre 15-19 anos com 21,28\% (5090), 30-39 anos com 23,10\%(5525), 40-49 anos com 14,14\%(3381), 50-59 anos com 6,53\%(1561) e mais de 60 anos com 6,50\%(1555). Resultado semelhante foi verificado em estudo conduzido no Rio Grande - RS onde se verificou que a faixa etária mais acometida entre mulheres vítima de violência foi entre 20-29 anos seguida da faixa entre 30-39 anos (ACOSTA et al., 2013).

Do total de mulheres que sofreram algum tipo de violência, 68,85\% (15396) pertenciam à cor/raça branca, seguido da cor/raça parda com 19,15\% (4282) e negra com 5,69\% (1273). Resultado semelhante foi observado em estudo com 902 casos de violência registrados em ocorrência policial em Rio Grande-RS (ACOSTA et al., 2013). Em contrapartida, GARCIA et al. (2016) realizaram inquérito em 24 capitais brasileiras e no Distrito Federal e verificaram que $62,6 \%$ das mulheres vítimas de agressão eram da cor/raça negra/parda.

Em relação à escolaridade verifica-se que a violência ocorre mais em mulheres com escolaridade inferior a oito anos com 41,52\% (9284) (Tabela 1)

TABELA 1. Distribuição das notificações de violência contra a mulher segundo escolaridade, 2009-2014, Paraná.

Escolaridade

\begin{tabular}{lrr}
\hline Sem escolaridade & 364 & 1,63 \\
Ensino fundamental & 9284 & 41,52 \\
Ensino médio & 6400 & 28,62 \\
Ensino superior & 1404 & 6,28 \\
Ignorado/branco & 4910 & 21,96 \\
\hline Total & $\mathbf{2 2 3 6 2}$ & $\mathbf{1 0 0}$
\end{tabular}

Fonte: SINAN, (2015).

\section{$\mathbf{N}$}

22362

Mulheres de baixa escolaridade estão mais vulneráveis a ocorrência de violência. Esse dado foi corroborado por outros estudos onde se verificou que a proporção de mulheres vítimas desse agravo possuem escolaridade inferior a oito anos de estudo (SCHRAIBER et al., 2002; GARCIA et al., 2016).

O local de ocorrência mais frequente foi a residência com 69,50\% (15542), seguido da via pública com 16,75\% (3745). Do total de notificações, 46,37\% (10370) das mulheres agredidas já haviam sofrido algum tipo de violência anteriormente. Estudo realizado em Bélem-PA entre 2009-2011 verificou 3267 casos de violência contra a mulher nesse período e a residência das vítimas foi o local mais comum de ocorrência com um total de 74\%, seguida da via pública com 11,3\% (VELOSO et al., 2013).

Em relação ao tipo de agravo mais frequente, 48,05\% (16861) sofreram violência física, seguido de violência psicológica ou moral com 29,40\% (10316) (Figura 2). Pesquisas evidenciaram que a violência física e a psicológica são as formas mais recorrentes (LETTIERE \& NAKANO, 2011; SILVA et al., 2013; GARCIA et al., 2016). Estudo realizado em município do Rio Grande - RS com dados da Delegacia de Polícia Especializada no Atendimento às Mulheres verificou nas ocorrências registradas predomínio de violência física $(47,7 \%)$ seguida da violência psicológica (44,1\%) (SILVA et al., 2013). 
Estudo conduzido em Ribeirão Preto com 265 usuárias da atenção básica com o objetivo de verificar a prevalência de violência entre as mulheres encontrou que a violência psicológica ocorreu pelo menos uma vez na vida para $41,5 \%$ das entrevistadas, violência física para $26,4 \%$ e violência sexual para $9,8 \%$ (MARINHEIRO et al., 2006).

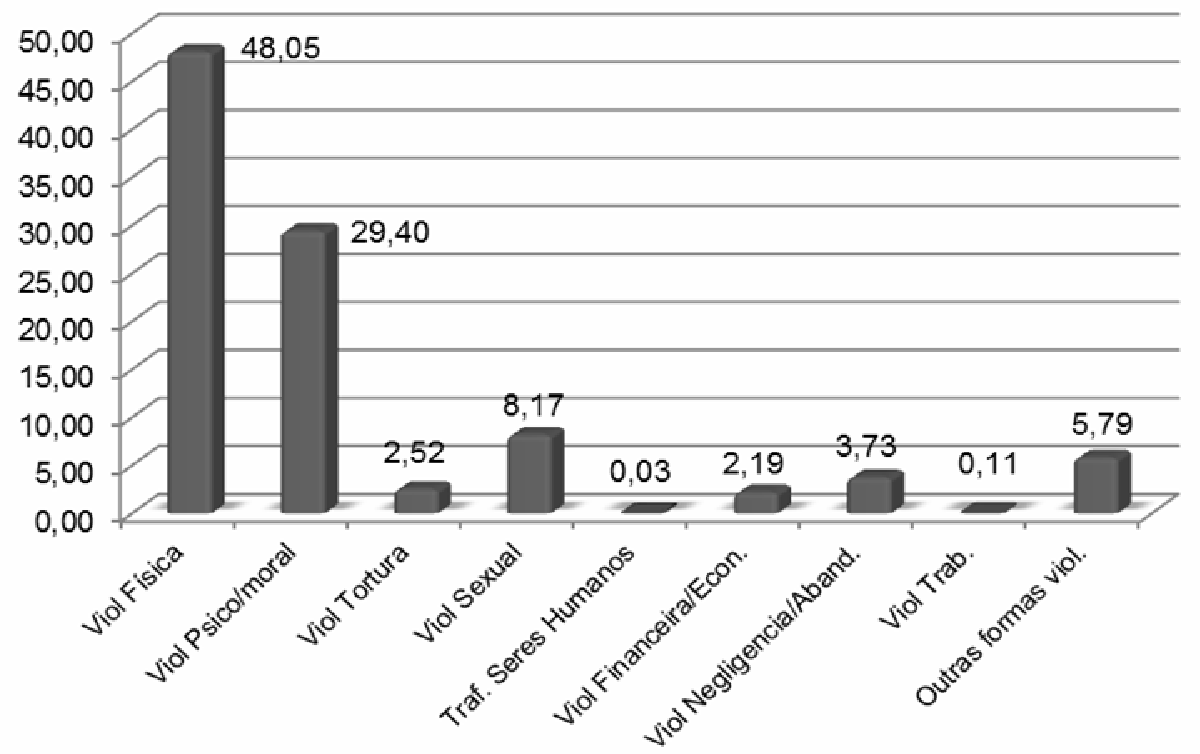

FIGURA 2. Distribuição percentual das notificações de violência contra a mulher no Paraná segundo tipo de violência, 2009-2014. Fonte: SINAN, (2015).

O meio de agressão mais prevalente foi o espancamento com 46,04\% (13389), seguido de ameaça $21,22 \%$ (6170) e estupro com 7,62\% (2217) (Figura 3).

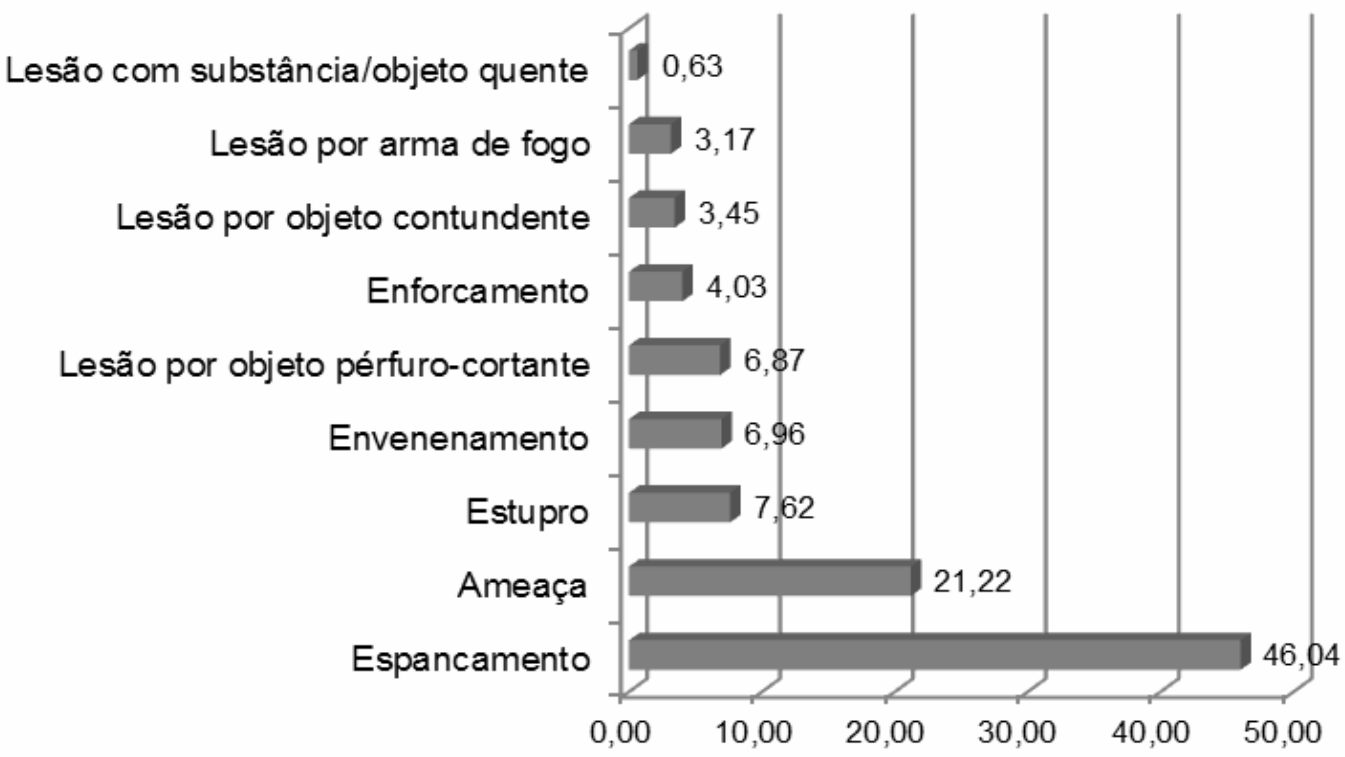

FIGURA 3. Distribuição das notificações de violência segundo meio de agressão, Paraná 2009-2014. Fonte: SINAN, (2015). 
Estudo realizado em Bélem-PA com dados de notificação de violência verificou que o meio de agressão mais comum foi ameaça com $59,7 \%$, seguido de espancamento com $27,4 \%$ (VELOSO et al., 2013). Estudo realizado nas capitais brasileiras verificou que o meio mais comum foi o espancamento com $60,7 \%$, seguido de lesão por objeto perfuro-cortante (GARCIA et al., 2016).

A violência sexual mesmo em proporções inferiores a outros tipos de violência causam um impacto substancial na vida dessas mulheres. De acordo com a OMS mulheres que sofreram violência sexual são 2,3 vezes mais propensas a ter transtornos por uso de álcool e 2,6 vezes mais propensas a sofrer de depressão ou ansiedade (WHO, 2013).

A Figura 4 apresenta que 30,96\% (7115) das mulheres foram vítimas de seu cônjuge. Destaca-se que a própria pessoa cometeu violência contra si em 13,35\% (3067) das notificações. De acordo com SCHRAIBER et al. (2002) muito frequentemente, o problema da violência traduz-se em diversas repercussões para a saúde das mulheres e sua qualidade de vida. A violência conjugal e o estupro têm sido associados a maiores índices de suicídio, abuso de drogas e álcool. Em último, observa-se o cuidador como agressor com $0,14 \%$ (33).

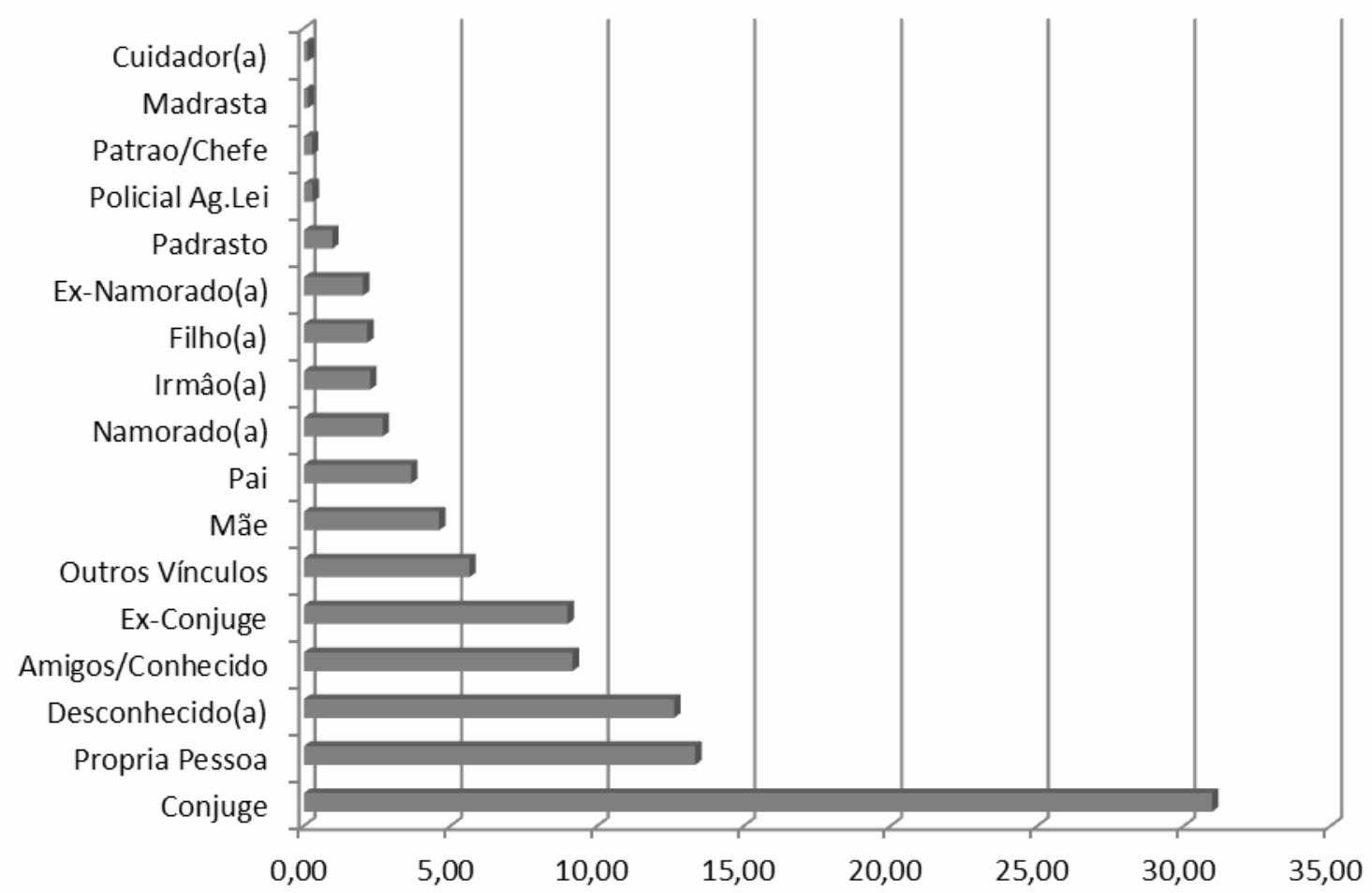

FIGURA 4. Distribuição das notificações de violência segundo agressor, Paraná 2009-2014. Fonte: SINAN, (2015).

De acordo com a OMS cerca de $35 \%$ das mulheres em todo o mundo têm sofrido tanto violência física e/ou sexual seja por parte do parceiro ou desconhecido. Embora existam muitas outras formas de violência que as mulheres possam estar expostas, esta já representa uma grande proporção de mulheres de todo o mundo (WHO, 2013).

Após a ocorrência da violência, o encaminhamento dado foi ambulatorial em $56,64 \%$ (12666) dos casos e hospitalar em $14,11 \%$ (3155). O desfecho foi alta em 83,36\% (18642) dos casos, óbito em decorrência da violência em 1,07\% (239) e 
evasão ou fuga em 1,44\% (322). GARCIA et al. (2016) verificaram que após a alta o desfecho mais comum foi internação hospitalar seguido de encaminhamento ambulatorial.

\section{CONCLUSÃO}

Os resultados observados neste estudo são de grande relevância. Conhecer o perfil epidemiológico das notificações de violência contra a mulher no Paraná permitiu assegurar que este é de fato um crescente problema da saúde coletiva, sua magnitude evidencia a desigualdade de gênero e a emergente necessidade da elaboração de políticas públicas para redução, controle do problema, proteção das mulheres em situação de vulnerabilidade e promoção da saúde e qualidade de vida dessas. Dessa forma, faz-se necessário, cada vez mais, a veiculação de políticas que visem a conscientização da população sobre esse assunto. As repercussões desse problema vão além da saúde física e mental, se estendendo para a convivência entre familiares, principalmente quando o agressor e a vítima moram juntos e com mais integrantes da família, como filhos, sogros, sobrinhos etc. Contribuindo, assim, para intensificação dos problemas entre familiares e problemas sociais. Os resultados desse estudo permitiram direcionar um olhar ao lamentável e frequente problema que a sociedade precisa enfrentar. Busca-se a divulgação dessas informações para melhor compreensão desse evento e que a busca de soluções para sua resolução seja prioritária para que as mulheres deixem de sofrer com esse agravo. Recomenda-se ainda a realização de novos estudos e que estes sejam realizados em outros estados, pois a violência pode apresentar padrão de ocorrência diferenciado levando em consideração diferenças na aceitação cultural da violência contra a mulher.

\section{REFERÊNCIAS}

ACOSTA, D. F.; GOMES, V. L. O.; BARLEM, E. L. D. Perfil das ocorrências policiais de violência contra a mulher. Acta Paulista de Enfermagem, v. 26, n. 6, p. 547-553, 2013. Disponível em: http://www2.unifesp.br/acta/pdf/v26/n6/v26n6a7.pdf

BRASIL. Lei $n$ o 11.340, de 7 de agosto de 2006. Cria mecanismos para coibir a violência doméstica e familiar contra a mulher, nos termos do $\S 80$ do art. 226 da Constituição Federal, da Convenção sobre a Eliminação de Todas as Formas de Discriminação contra as Mulheres e da Convenção Interamericana para Prevenir, Punir e Erradicar a Violência contra a Mulher; dispõe sobre a criação dos Juizados de Violência Doméstica e Familiar contra a Mulher; altera o Código de Processo Penal, o Código Penal e a Lei de Execução Penal; e dá outras providências. Diário Oficial da república Federativa do Brasil. Brasília, DF, 07 ago. 2006. Disponível em: http://www.planalto.gov.br/ccivil_03/_ato2004-2006/2006/lei/111340.htm.

GARCIA, L. P.; DUARTE, E. C.; FREITAS, L. R. S.; SILVA, G. D. M. Violência doméstica e familiar contra a mulher: estudo de casos e controles com vítimas atendidas em serviços de urgência e emergência. Caderno de Saúde Pública, Rio de Janeiro, v. 32, n. 4, 2016. Disponível em: http://www.scielo.br/scielo.php?script=sci_arttext\&pid=S0102-

311X2016000400704\&lng=en\&nrm=iso. $\quad$ DOI: http://dx.doi.org/10.1590/0102$311 \times 00011415$. 
IPEA. Avaliando a efetividade da Lei Maria da Penha. Texto para discussão/Instituto de Pesquisa Econômica Aplicada.- Brasília : Rio de Janeiro, 2015.

Disponível

em:

http://www.ipea.gov.br/portal/images/stories/PDFs/TDs/td_2048.pdf

LETTIERE, A.; NAKANO, A. M. S. Violência doméstica: as possibilidades e os limites de enfrentamento. Revista Latino Americana de Enfermagem, Ribeirão Preto, v. 19, n. 6, p. 1421-1428, 2011. Disponível em: http://www.scielo.br/scielo.php?script=sci_arttext\&pid=S0104-

11692011000600020\&lng=en\&nrm=iso DOI: http://dx.doi.org/10.1590/S010411692011000600020.

LUCENA, K. D. T.; SILVA, A. T. M. C.; MORAES, R. M.; SILVA, C. C.; BEZERRA, I. M. P. Análise espacial da violência doméstica contra a mulher entre os anos de 2002 e 2005 em João Pessoa, Paraíba, Brasil. Caderno de Saúde Pública, Rio de Janeiro, v. 28, n. 6, p. 1111-1121, 2012. Disponível em: http://www.scielosp.org/scielo.php?script=sci_arttext\&pid=S0102-

311X2012000600010\&Ing=en\&nrm=iso. $\quad$ DOI: $\quad$ http://dx.doi.org/10.1590/S0102$311 \times 2012000600010$.

MARINHEIRO, A. L. V.; VIEIRA, E. M.; SOUZA, L. Prevalência da violência contra a mulher usuária de serviço de saúde. Revista Saúde Pública, v. 40, n. 4, p. 604-10, 2006. Disponível em: http://www.scielo.br/pdf/rsp/nahead/ao-5008.pdf.

SCHRAIBER, L. B.; D'OLIVEIRA, A. F. P. L.; FRANCA-JUNIOR, I.; PINHO, A. A. Violência contra a mulher: estudo em uma unidade de atenção primária à saúde. Revista Saúde Pública, v. 36, n. 4, p. 470-7, 2002. Disponível em: http://www.scielosp.org/pdf/rsp/v36n4/11766.

SILVA, C. D.; GOMES, V. L. O.; ACOSTA, D. F.; BARLEM, E. L. D.; FONSECA, A. D. Epidemiologia da violência contra a mulher: características do agressor e do ato violento. Journal of Nursing UFPE/Revista de Enfermagem UFPE, v. 7, n. 1, 2013. Disponível em:

http://www.revista.ufpe.br/revistaenfermagem/index.php/revista/article/viewFile/3554/ pdf_1791. DOI: 10.5205/reuol.3049-24704-1-LE.0701201302.

SILVA, L. E. L.; OLIVEIRA, M. L. C. Características epidemiológicas da violência contra a mulher no Distrito Federal, 2009 a 2012. Epidemiologia e Serviços de Saúde, Brasília, v. 25, n. 2, p. 331-342, 2016. Disponível em http://scielo.iec.pa.gov.br/scielo.php?script=sci_arttext\&pid=S1679-

49742016000200331\&lng=pt\&nrm=iso. DOI: http://dx.doi.org/10.5123/S167949742016000200012.

VEloso, M. M. X.; MAGALHÃES, C. M. C.; DELL'AGLIO, D. D.; CABRAL, I. R.; GOMES, M. M. Notificação da violência como estratégia de vigilância em saúde: perfil de uma metrópole do Brasil. Ciência e saúde coletiva, Rio de Janeiro, v. 18, n. $5, \quad$ p. 1263-1272, 2013. Disponível em: http://www.scielo.br/scielo.php?script=sci_arttext\&pid=S1413- 
81232013000500011\&lng=en\&nrm=iso. $\quad$ DOI: $\quad$ http://dx.doi.org/10.1590/S141381232013000500011.

WHO. Global and regional estimates of violence against women: prevalence and health effects of intimate partner violence and non-partner sexual violence. World Health Organization, 2013. Disponível em: http://apps.who.int/iris/bitstream/10665/85239/1/9789241564625_eng.pdf 